\title{
The use of whole body diffusion-weighted post-mortem magnetic resonance imaging in timing of perinatal deaths
}

\author{
Susan C. Shelmerdine ${ }^{1,2}$ (D) Cheryl Main ${ }^{3} \cdot$ John Ciaran Hutchinson ${ }^{1,2} \cdot$ Dean Langan $^{2} \cdot$ Neil J. Sebire $^{1,2}$. \\ Owen J. Arthurs ${ }^{1,2}$
}

Received: 7 November 2017 / Accepted: 19 July 2018 / Published online: 28 July 2018

(C) The Author(s) 2018

\begin{abstract}
Objectives Diffusion-weighted MRI provides information regarding body water movement following death, which may be an imaging marker of post-mortem interval (time since death; PMI) or maceration (degree of tissue degradation during intra-uterine retention) in perinatal deaths. Our aim was to evaluate the relationship between maceration, PMI and body organ apparent diffusion coefficient (ADC) values in a cohort of subjects across a wide gestational range.

Materials Whole body post-mortem MRI with diffusion-weighted imaging (DWI) sequences were performed at $1.5 \mathrm{~T}$, with $b$ values of 0,500 and $1000 \mathrm{~mm}^{2} / \mathrm{s}$. Mean ADC values were calculated from regions of interest (ROIs) placed in the lungs, myocardium, spleen, renal cortex, liver and psoas muscle by two independent readers. Multivariable regression analysis was performed against PMI, gestational age, post-mortem weight, maceration score and gender.

Results Eighty perinatal deaths were imaged with mean gestational age of 32 weeks (18-41 weeks), of which 49 (61.3\%) were male. The mean PMI was 8 days (1-18 days). Maceration scores were statistically significant predictive factors for ADC values in all included body organs except the lungs, but PMI was not a predictor for ADC values in any body organ. In the absence of maceration $(n=14)$, PMI was not statistically associated with ADC values in any of the body areas. The ratio of agreement in the majority of body areas was close to 1 (range between 0.95 and 1.10).

Conclusion Maceration, not PMI, is significantly associated with ADC values in perinatal deaths. Further research is needed to understand organ-specific changes in the post-mortem period.
\end{abstract}

Keywords Autopsy $\cdot$ Post-mortem $\cdot$ MRI $\cdot$ Diffusion $\cdot$ Perinatal $\cdot$ Paediatric

Susan C. Shelmerdine

Susan.shelmerdine@gosh.nhs.uk

Cheryl Main

Cherylmain@doctors.org.uk

John Ciaran Hutchinson

c.hutchinson@ucl.ac.uk

Dean Langan

d.langan@ucl.ac.uk

Neil J. Sebire

Neil.sebire@gosh.nhs.uk

Owen J. Arthurs

Owen.arthurs@gosh.nhs.uk

1 Great Ormond Street Hospital for Children NHS Foundation Trust, London WC1N 3JH, UK

2 UCL Great Ormond Street Institute of Child Health, 30 Guilford Road, London WC1N 1EH, UK

3 University Hospital Southampton NHS Foundation Trust, Southampton SO16 6YD, UK

\author{
Abbreviations \\ ADC Apparent diffusion coefficient \\ DWI Diffusion-weighted (magnetic resonance) \\ imaging \\ (PM)MR (Post-mortem) magnetic resonance imaging \\ PMI Post-mortem interval \\ ROI Region of interest
}

\section{Introduction}

Post-mortem magnetic resonance imaging (PMMR) has been shown to have a high diagnostic accuracy in identification of perinatal abnormalities and cause of death [1, 2]. Diffusionweighted imaging (DWI), a sequence that is used in PMMR imaging protocols [3], derives contrast from the differences in movement of water molecules within various tissues [4] and can be indirectly quantified using the apparent diffusion coefficient (ADC) [5]. This value can provide information relating to the 
microscopic structure and functional changes at a cellular level within body organs where structures with high cellularity (i.e. causing restriction of movement of water molecules) demonstrate low $\mathrm{ADC}$ values, compared to less cellular structures with higher ADC values [6]. In live children, this imaging sequence has shown to have promising clinical uses, such as for the assessment of tumour response to treatment [7] and in the identification of inflammatory changes (such as in inflammatory bowel disease [8] and enthesitis-related arthritis [9]).

The application of DWI in the post-mortem setting is appealing as it (a) does not require the administration of an exogenous contrast agent, (b) the irreversible and progressive cellular changes that occur as a consequence of autolysis should be detectable and (c) could result in ADC values that change predictably over time, and may allow the estimation of time since death, or post-mortem interval (PMI). Nevertheless, imaging assessment of perinatal post-mortem tissue has several confounders. In addition to autolysis (i.e. the enzymatic degradation of tissue following death), foetuses which have suffered intra-uterine demise and retention prior to delivery undergo a process of maceration (i.e. tissue degeneration within a fluid-filled cavity (amniotic fluid)). Figure 1 provides a diagrammatical representation of the relative timing of these processes. Given that exact knowledge of an intra-uterine death is frequently unknown (and thus the timing of intra-uterine retention inaccurate), the degree of maceration at autopsy by external assessment is commonly used as a subjective, surrogate marker to estimate intra-uterine retention time [10].

Reports of PMMR imaging in the medical literature which have used DWI have shown that in the foetal brain (specifically the basal ganglia) [11] and lung [12] the ADC value changes are associated with PMI. Maceration was also found to affect ADC values in most areas of the foetal brain, but other body organs have not been comprehensively evaluated.

The aim of this study was therefore to evaluate the relationship between maceration, PMI and body organ ADC values across a wide gestational age range in the setting of perinatal death. If the $\mathrm{ADC}$ values in body organs are found to be robust marker of PMI or maceration, this could have implications for the investigation of intra-uterine foetal deaths, especially where timing may be medico-legally important.

\section{Methods}

\section{Study cohort}

Written informed consent was obtained from all parents of participants for clinical pre-autopsy PMMR, as part of our institution's post-mortem imaging protocol. DWI sequences were prospectively performed on all post-mortem foetal, stillbirth and early neonatal deaths referred to our institution from
May 2013 to September 2016 (40 months). Cases in which the DWI imaging was of inadequate quality, incomplete, or where there was significant organ abnormality on imaging or at autopsy were excluded. Bodies were stored in the mortuary at $4{ }^{\circ} \mathrm{C}$ within $24 \mathrm{~h}$ of delivery and PMMR was performed during dedicated research imaging lists to prevent any disruption to usual clinical services.

Demographic included age at time of death (for neonatal deaths, in days), gestational age (in weeks) and post-mortem interval (PMI, days from delivery to imaging). Intra-uterine retention interval (time between foetal death and delivery/extraction) was not recorded due to unreliable timing and inconsistent documentation in the notes. For this reason, we used maceration score (scored subjectively by the duty pathologist: 0-none, 1-mild or moderate, 2-established [10]).

\section{Magnetic resonance imaging}

All MR imaging was performed at $1.5 \mathrm{~T}$ (Avanto, Siemens Medical Solutions, Erlangen, Germany) with a conventional phase array body coil. Body imaging included isovolumetric T1- and T2-weighted imaging for the whole body (neck to feet) with additional isovolumetric T2-weighted constructive interface stead state (CISS) sequences covering the thorax (for cardiac anomalies) and a 2D axial T2-weighted inversion recovery sequence of the body, according to published protocols [3]. The coil used was dependent on patient size, selected to allow for best image quality.

Diffusion-weighted imaging sequences were performed using single-shot spin echo-planar imaging (EPI) in the axial plane, with the following parameters: 19 slices in three noncollinear axis directions, EPI factor 95, TR $2700 \mathrm{~ms}$; TE $96 \mathrm{~ms}$; FOV $230 \mathrm{~mm} ; 128 \times 128$ matrix, 5-mm-slice thickness with $1-\mathrm{mm}$ gap, acquisition time $90 \mathrm{~s}$. Diffusion gradient values were $b=0,500$ and $1000 \mathrm{~s} / \mathrm{mm}^{2}$.

\section{DWI analysis}

From the native DWI acquisition, ADC maps were obtained on the acquisition console (Syngo, Siemens Medical Solutions, Erlangen, Germany). Data was then transferred to Osirix (open source DiCOM viewer: http://www.osirixviewer.com) for $\mathrm{ADC}$ measurements in different regions of interest (ROI). Circular ROIs were drawn manually and plotted on ADC maps for all cases by two independent readers blinded to one another's findings and also to the post-mortem report. Where there was difficulty in identifying the delineation of the target organ, cross-referencing was made to the isovolumetric $\mathrm{T} 2$ sequences obtained during the same MRI examination for anatomical information. The readers read the cases once. Both readers were qualified paediatric radiologists with 7 years experience each. 
Fig. 1 Illustration of the timing of events where intra-uterine foetal demise has occurred, to highlight the terms 'post-mortem interval (PMI)' and intra-uterine death interval. The degradation of tissue via autolysis (green arrow) occurs after the death of the foetus, whereas those of maceration (red arrow) occur only whilst the foetus remains in utero. In cases where the foetus is born alive and subsequently dies, maceration by definition will not have occurred and the body will only undergo autolysis

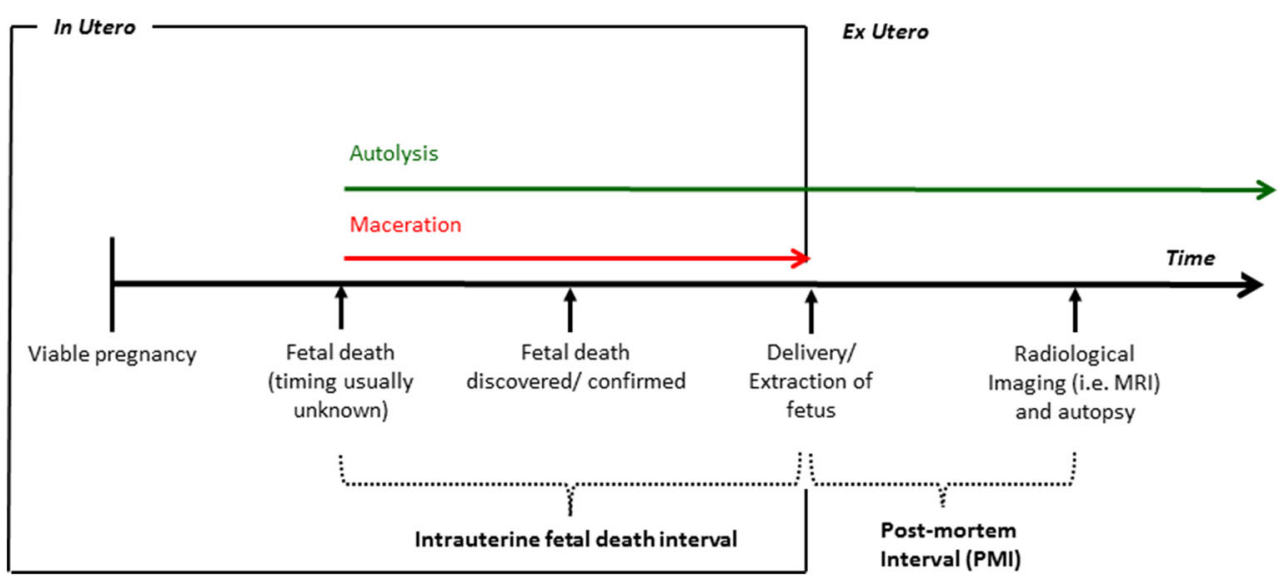

The ROIs were placed on the following body locations (Fig. 2):

1) Lung: obtained within the largest region of 'normal' lung parenchyma (i.e. non-consolidated regions for neonatal deaths, and for all cases - not including any pleural effusions).

2) Myocardium: within the intraventricular septum or left ventricular muscle, whichever allowed the largest ROI volume to be obtained.

3) Right renal cortex: avoiding any renal pelvis.

4) Liver: within the right lobe of the liver to obtain the most representative ROI sample.

5) Spleen: within the centre of the organ to include the largest ROI possible

6) Right psoas muscle: used as a control value.
For each ROI, a mean ADC value $\left(\times 10^{-5} \mathrm{~mm}^{2} / \mathrm{s}\right) \pm$ standard deviation (SD) was obtained. The circular area for the ROI was drawn to include as much of the target organ as possible, whilst maintaining a relative $\mathrm{SD}<10 \%$ of the mean to ensure homogeneity, typically $<25 \mathrm{~mm}^{2}$. Size-specific ROIs were used for each individual case rather than choosing a fixed ROI, due to the range of foetal sizes (weight and gestation range given in Table 1) in our sample size. Where a valid ADC value for an organ could not be obtained (either due to a small organ inclusion area on the ADC map or significant artefact), then this was left blank or unrecorded.

Temperature correction for ADC values was not applied given that our sample included only post-mortem cases, imaged and stored at comparable temperatures. No comparison or extrapolation of our values with live cases was performed.
Fig. 2 Foetal thoraco-abdominal post-mortem axial apparent diffusion coefficient (ADC) maps from DWI sequences $(\mathbf{a}-\mathbf{d})$ in a 32 gestational age foetus. The axial slices illustrate the lungs and myocardium (a), liver and spleen (b), kidneys (c) and psoas muscle (d) with corresponding regions of interest drawn
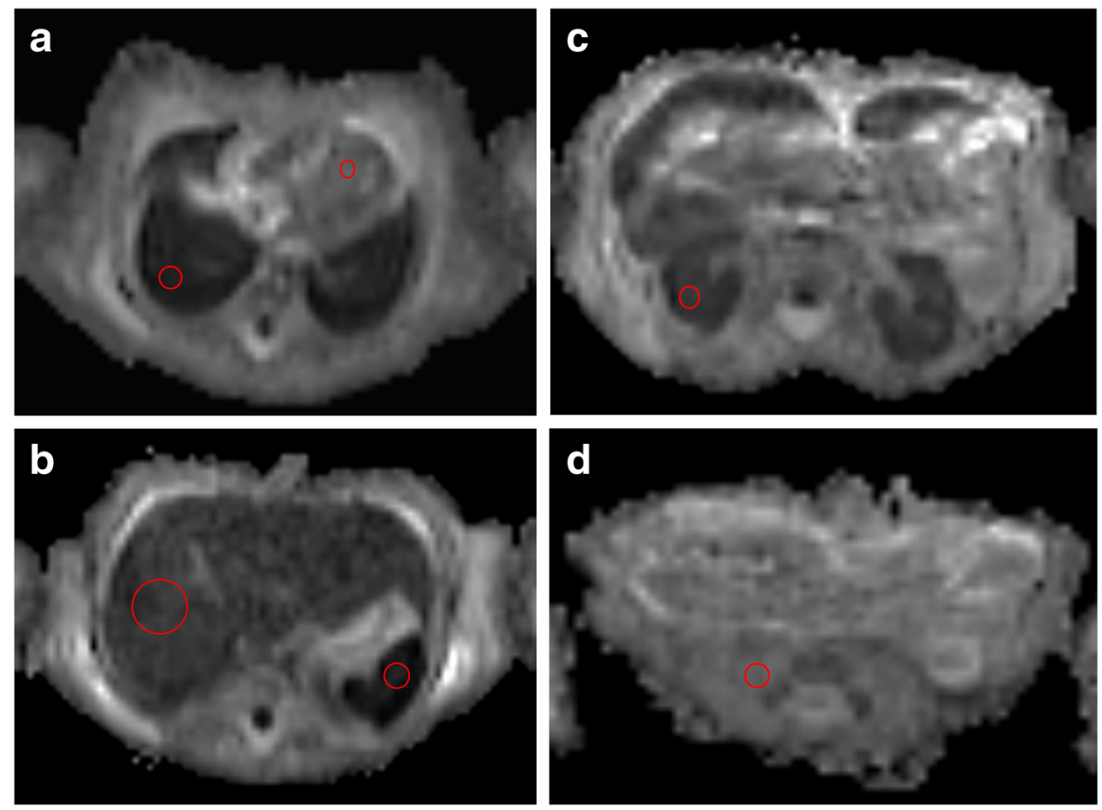
Table 1 Demographic data of the 80 perinatal deaths included in this study. The neonatal subgroup $(n=14)$ were delivered alive but died within the first month of life.

\begin{tabular}{llllllr}
\hline & Case group & $N$ & Mean & Median & IQR & Range \\
\hline $\begin{array}{l}\text { Age at death (days) } \\
\begin{array}{l}\text { Gestational age } \\
\quad \text { (weeks) }\end{array}\end{array}$ & $\begin{array}{l}\text { Neonatal deaths } \\
\text { Neonatal deaths }\end{array}$ & 14 & 7 & 1 & $0,14.8$ & $0-25$ \\
$\begin{array}{c}\text { Gestational age } \\
\quad \text { (weeks) }\end{array}$ & $\begin{array}{c}\text { Stillbirths, miscarriages and } \\
\text { terminations* }\end{array}$ & 66 & 32 & 34 & 27,38 & $18-41$ \\
$\begin{array}{c}\text { Post-mortem weight } \\
\text { (kg) }\end{array}$ & All cases & 80 & 2.06 & 2.292 & $\begin{array}{c}0.783, \\
3.006\end{array}$ & $0.258-4.73$ \\
$\begin{array}{c}\text { Post-mortem interval } \\
\text { (days) }\end{array}$ & All cases & 80 & 8 & 7.5 & 5,10 & $1-18$ \\
\hline
\end{tabular}

*The remainder of our cases which were not neonatal deaths were born without signs of life. Their date of death and date of birth are the same and age at death is therefore 0 days

\section{Statistical analysis}

Multilevel models were fitted for each ROI with mean corrected ADC values $\left(10^{-5} \mathrm{~mm}^{2} / \mathrm{s}\right)$ with the outcome variable subject and reader both included as complete cross-classified random intercepts. Fixed effect predictor variables include maceration scores (MAC), post-mortem interval (PMI), gestational age, gender and post-mortem weight. Given a prior publication stating maceration to be a strongest predictor in ADC values for post-mortem foetal brain [11], we extracted a sub-group of cases in whom maceration could not have occurred (i.e. neonatal deaths; babies who were born alive and subsequently died). The mean corrected ADC values for each ROI were plotted against the post-mortem interval and the adjusted $R^{2}$ estimates were calculated to summarise the strength of any linear relationships for the various body organs. This subsample contained 14 patients, and therefore given the low sample size, it was not possible to adjust for multiple predictors.

To measure inter-reader variability, Bland-Altman plots were constructed with $95 \%$ limits of agreement on the ratio between the two reader mean ADC values. The ratios of the two measurements were calculated, instead of the absolute difference, because agreement is dependent on the scale of the ADC measurement. All analyses were carried out in R version 3.3.0 and the $\mathrm{R}$ package lme4 version 1.1.141 was used for multilevel analysis.

\section{Results}

\section{Study sample}

We identified $80(49,61.3 \%$ male $)$ perinatal deaths for inclusion in the study: $14(17.5 \%)$ neonatal deaths, $21(26.2 \%)$ termination of pregnancies (TOP), 2 (2.5\%) miscarriages and $43(53.8 \%)$ stillbirth/intra-uterine deaths.
The patient demographics are given in Table 1. Table 2 lists the mean ADC values for the different body areas based on the total number of readings obtained by both readers.

\section{Multivariate analysis}

Using multilevel modelling, six prediction models were fitted for the lungs, myocardium, renal cortex, liver, spleen and psoas muscle. Maceration score was a statistically significant predictor for ADC values in all body areas except the lungs (Table 3). The other five models predict an increase in ADC values of between 93.82 (spleen, 95\% CI 31.19-156.4) and 321.8 (myocardium, 95\% CI 230.1-413.5) in patients with a maceration score of 2 (in comparison with maceration score 0 ). Post-mortem interval (PMI) was not a predictor for ADC values for any of the body organs after adjusting for all other predictor variables.

\section{Non-macerated perinatal deaths}

In order to assess the relative contribution of maceration, we identified 14 neonatal deaths ( 7 males; 50\%) which by definition had not undergone maceration. This sub-group had median PMI of 5 days (range 1-12 days) with median PM weight of $2.625 \mathrm{~kg}$ (range $0.339-4.335 \mathrm{~kg}$ ). PMI was not a significant predictor of ADC values in any body area in this subsample.

\section{Reproducibility}

Readers had the highest agreement in the right psoas muscle, with mean ratio of 1.04 and $95 \%$ limits of agreement (LOA) of the ratios between 0.80 and 1.35. Agreement was poorest in the spleen, with mean ratio of 1.02 and $95 \%$ LOA between 0.42 and 2.44. The mean ratio of agreement in all body areas was close to 1 (range between 0.95 and 1.10). 
Table 2 Summary of body organ ADC measurements $\left(\times 10^{-5} \mathrm{~mm}^{2}\right)$ $\mathrm{s})$ in all 80 perinatal death cases, by both readers. The $N$ numbers states the number of observations that the mean and range ADC values are derived from (i.e. if both readers could obtain an ADC score for all cases, then $N=160$ )

\begin{tabular}{lllllll}
\hline & $N$ (total no. observations) & Median & Mean & Range & IQR & SD \\
\hline Lung ADC & 145 & 431 & 448 & $171-1073$ & 375,514 & 45 \\
Myocardium ADC & 136 & 610 & 615 & $224-1231$ & 399,783 & 58 \\
Right renal cortex ADC & 160 & 422 & 454 & $195-1046$ & 315,531 & 48 \\
Liver ADC & 159 & 450 & 439 & $66-829$ & 279,581 & 50 \\
Spleen ADC & 148 & 276 & 329 & $66-857$ & 221,386 & 64 \\
Right psoas ADC & 159 & 585 & 594 & $352-1069$ & 486,687 & 65 \\
\hline
\end{tabular}

\section{Discussion}

This study found that the maceration score but not PMI was significantly associated with post-mortem perinatal ADC body organ values. Caution should therefore be exercised when interpreting DWI PMMR to avoid attributing ADC changes to post-mortem interval.

The association of maceration with ADC values is supported in part by a previous study assessing post-mortem foetal brain ADC values, where this played a role in all regions of the brain analysed. Maceration is usually assessed at autopsy by external appearances, including skin changes, umbilical cord discoloration and cranial collapse, but the underlying cellular process remains poorly understood [10]. Changes in membrane permeability, redistribution of magnetization between compartments and increased diffusivity within the remaining extracellular space may all account for the diffusivity changes observed in our study [13] giving differences in ADC values. Clearly, a combination of autolysis and maceration contribute to the changes seen in this study.

When we analysed a subset of non-macerated foetuses, we did not find an underlying effect of post-mortem interval on ADC changes. Whilst previous studies in perinatal deaths have not been performed, a recently published study of 19 adult post-mortem cases [14] found that the ADC values of the liver did not significantly change with time (in hours) since death, with temperature changes accounting for a greater effect. Nevertheless, the ADC values in this study were measured at two hourly intervals over $16 \mathrm{~h}$, and this may not have been enough time for effects to be observed.

Previous work performed for a set of 15 foetuses and neonates reported that ADC changes in the lung may be correlated to PMI [12]; however, in our larger cohort, we could not reproduce those findings. Of the 15 subjects that were analysed in the comparator study, the majority $(n=10)$ were estimated to have undergone in-utero retention ranging from between 0.5 and 2 days prior to delivery, with 4 subjects allocated a maceration score of $\geq 1$ at autopsy. Due to the small sample size, neonatal deaths were not compared to intra-uterine deaths and the reported changes in lung ADC values with time may have therefore been a property of underlying maceration and autolysis rather than post-mortem interval.
Although we have assessed the use of ADC values in the largest perinatal population studied to date, our study has several limitations, with relatively small sample size and only fair inter-reader reproducibility. We speculate that this may be due to inter-observer variation in ROI creation, and limited experience in analysing post-mortem DWI imaging. We also recognise that the use of size-specific ROIs may have generated further variation, but a fixed size ROI would not have yielded accurate results across the range of foetal weights and gestations in our sample size. There may have been some restrictions in our technique, as all of our cases were imaged at $1.5 \mathrm{~T}$ and not $3 \mathrm{~T}$. A higher magnetic field strength could have yielded greater image contrast for the anatomical referencing on isovolumetric $\mathrm{T} 2$ sequences to aid placement of body organ ROIs [15]; however, the accuracy of ADC values on high-field-strength MRI in the postmortem setting has yet to be evaluated, and may be more susceptible to field inhomogeneity and artefacts [16]. In addition, whilst we used standard $b$ values at 0,500 and $1000 \mathrm{~s} / \mathrm{mm}^{2}$, these may not be optimal for post-mortem imaging at low temperatures and truly quantitative imaging across this range could be useful in this setting. Nevertheless, our imaging techniques were standardised across all our subjects in this study following the same MRI protocol. We did not apply temperature correction to our data as the correlations and multivariate analysis would have been unaffected and we were not comparing our data with live subjects.

In addition, the usage of a maceration score is subjective, and higher scores do not always correlate with longer intra-uterine retention times [17]. Ideally, investigating the true effect of maceration would require knowing precisely the intra-uterine retention period. This is challenging in most intra-uterine deaths where the exact time of death is unknown (Fig. 1), but this may be possible following feticide for termination of pregnancy which could be assessed in future work. Equally, a larger subset of nonmacerated cases could be investigated in more detail. It is clear that the cellular changes that are detected by DWI are complex and related to several factors, and thus we urge caution if considering using these features in medicolegal and forensic cases. 
Table 3 Stepwise multivariate linear models. Multivariate linear regression, using potential predictors of reader, maceration score, gestational age, gender, postmortem (PM) weight and postmortem interval (PMI) in each model. Statistically significant results are highlighted in bold and in italics

\begin{tabular}{|c|c|c|c|c|c|c|}
\hline \multirow[t]{2}{*}{ Dependent variable } & \multirow[t]{2}{*}{ Independent variables } & \multirow[t]{2}{*}{$B$} & \multirow[t]{2}{*}{ s.e. } & \multicolumn{2}{|c|}{$95 \% \mathrm{CI}$ for B } & \multirow[t]{2}{*}{$p$ value } \\
\hline & & & & Lower & Upper & \\
\hline \multirow[t]{7}{*}{ Lung } & (Constant) & 417.2 & 128.1 & - & - & - \\
\hline & Maceration score $1(\mathrm{ref}=0)$ & 30.04 & 42.76 & -53.78 & 113.9 & 0.485 \\
\hline & Maceration score $2(\mathrm{ref}=0)$ & 11.13 & 30.86 & -49.36 & 71.61 & 0.720 \\
\hline & Gestational age (weeks) & -1.363 & 4.996 & -11.16 & 8.429 & 0.786 \\
\hline & Gender $($ ref $=$ female $)$ & -3.037 & 26.64 & -55.25 & 49.18 & 0.910 \\
\hline & PM weight (kg) & 6.523 & 25.67 & -43.79 & 56.84 & 0.800 \\
\hline & PMI & 6.949 & 4.138 & -1.162 & 15.06 & 0.098 \\
\hline \multirow[t]{7}{*}{ Myocardium } & (Constant) & 400.2 & 193.2 & - & - & - \\
\hline & Maceration score $1($ ref $=0)$ & 247.6 & 65.97 & 118.3 & 376.9 & $<0.001$ \\
\hline & Maceration score $2(r e f=0)$ & 321.8 & 46.79 & 230.1 & 413.5 & $<0.001$ \\
\hline & Gestational age (weeks) & 3.674 & 7.536 & -11.1 & 18.44 & 0.628 \\
\hline & Gender $($ ref $=$ female $)$ & 0.618 & 40.91 & -79.56 & 80.8 & 0.988 \\
\hline & PM weight (kg) & -64.95 & 38.93 & -141.2 & 11.35 & 0.100 \\
\hline & PMI & 7.468 & 6.344 & -4.966 & 19.9 & 0.244 \\
\hline \multirow[t]{7}{*}{ Spleen } & (Constant) & 345.1 & 134.1 & - & - & - \\
\hline & Maceration score $1(\mathrm{ref}=0)$ & 69.42 & 42.34 & -13.57 & 152.4 & 0.106 \\
\hline & Maceration score $2(r e f=0)$ & 93.82 & 31.95 & 31.19 & 156.4 & 0.005 \\
\hline & Gestational age (weeks) & -0.814 & 5.127 & -10.86 & 9.234 & 0.874 \\
\hline & Gender $($ ref $=$ female $)$ & 19.7 & 27.62 & -34.43 & 73.83 & 0.478 \\
\hline & PM weight (kg) & -57.09 & 25.62 & -107.3 & -6.875 & 0.029 \\
\hline & PMI & 8.328 & 4.306 & -0.111 & 16.77 & 0.057 \\
\hline \multirow[t]{7}{*}{ Right renal cortex } & (Constant) & 486.5 & 180.1 & - & - & - \\
\hline & Maceration score $1(\mathrm{ref}=0)$ & 111.6 & 57.66 & -1.452 & 224.6 & 0.057 \\
\hline & Maceration score $2(r e f=0)$ & 153.5 & 43.24 & 68.72 & 238.2 & 0.001 \\
\hline & Gestational age (weeks) & -4.731 & 6.902 & -18.26 & 8.797 & 0.495 \\
\hline & Gender $($ ref = female $)$ & 31.01 & 37.31 & -42.12 & 104.1 & 0.409 \\
\hline & PM weight (kg) & -22.15 & 34.8 & -90.36 & 46.06 & 0.526 \\
\hline & PMI & 7.982 & 5.788 & -3.363 & 19.33 & 0.172 \\
\hline \multirow[t]{7}{*}{ Liver } & (Constant) & 489.5 & 154.5 & - & - & - \\
\hline & Maceration score $1(r e f=0)$ & 142.6 & 49.61 & 45.39 & 239.8 & 0.005 \\
\hline & Maceration score $2(r e f=0)$ & 209.9 & 37.25 & 136.9 & 282.9 & $<0.001$ \\
\hline & Gestational age (weeks) & -5.403 & 5.940 & -17.04 & 6.239 & 0.366 \\
\hline & Gender $($ ref $=$ female $)$ & -41.84 & 32.13 & -104.8 & 21.13 & 0.197 \\
\hline & PM weight (kg) & 1.241 & 29.93 & -57.43 & 59.91 & 0.967 \\
\hline & PMI & 4.468 & 5.000 & -5.333 & 14.27 & 0.375 \\
\hline \multirow[t]{7}{*}{ Right psoas } & (Constant) & 501.0 & 116.3 & - & - & - \\
\hline & Maceration score $1($ ref $=0)$ & 83.63 & 37.22 & 10.68 & 156.6 & 0.028 \\
\hline & Maceration score $2(r e f=0)$ & 161.3 & 27.91 & 106.6 & 216.0 & $<0.001$ \\
\hline & Gestational age (weeks) & 1.734 & 4.469 & -7.025 & 10.49 & 0.699 \\
\hline & Gender $($ ref $=$ female $)$ & 48.53 & 24.10 & 1.295 & 95.77 & 0.048 \\
\hline & PM weight (kg) & -35.18 & 22.56 & -79.39 & 9.034 & 0.123 \\
\hline & PMI & -0.187 & 3.741 & -7.520 & 7.146 & 0.960 \\
\hline
\end{tabular}




\section{Conclusion}

The degree of maceration plays an important role when interpreting the post-mortem body organ ADC values, but we did not find a significant correlation with PMI even in cases where maceration was excluded. Further research is needed to understand the organ-specific changes during maceration for potential timing of intra-uterine demise in cases of stillbirth.

Acknowledgements Preliminary versions of this work have been presented at the British Society for Paediatric Radiology (BSPR) Annual Conference, London, UK (2016), and at the European Society of Paediatric Radiology (ESPR), Davos, Switzerland (2017).

Funding sources SCS is supported by a RCUK/UKRI Innovation Fellowship and Medical Research Council (MRC) Clinical Research Training Fellowship (Grant Ref: MR/R00218/1). This award is jointly funded by the Royal College of Radiologists (RCR). NJS is supported by NIHR Senior Investigator award and OJA by NIHR Clinician Scientist fellowship award. NJS receives funding from the Great Ormond Street Hospital Children's Charity.

This work was undertaken at GOSH/ICH, UCLH/UCL who received a proportion of funding from the United Kingdom Department of Health's NIHR Biomedical Research Centre funding scheme.

This article presents independent research funded by the NIHR.

\section{Compliance with ethical standards}

Disclaimer The views expressed are those of the authors and not necessarily those of the NHS, the NIHR or the Department of Health.

Open Access This article is distributed under the terms of the Creative Commons Attribution 4.0 International License (http:// creativecommons.org/licenses/by/4.0/), which permits unrestricted use, distribution, and reproduction in any medium, provided you give appropriate credit to the original author(s) and the source, provide a link to the Creative Commons license, and indicate if changes were made.

\section{References}

1. Thayyil S, Sebire NJ, Chitty LS, Wade A, Chong W, Olsen O, Gunny RS, Offiah AC, Owens CM, Saunders DE, Scott RJ, Jones R, Norman W, Addison S, Bainbridge A, Cady EB, Vita ED, Robertson NJ, Taylor AM, group Mc (2013) Post-mortem MRI versus conventional autopsy in fetuses and children: a prospective validation study. Lancet 382(9888):223-233

2. Ashwin C, Hutchinson JC, Kang X, Langan D, Jones R, Norman W, Cannie M, Jani J, Sebire NJ, Arthurs OJ (2017) Learning effect on perinatal post-mortem magnetic resonance imaging reporting: single reporter diagnostic accuracy of 200 cases. Prenat Diagn 37(6):566-574
3. Norman W, Jawad N, Jones R, Taylor AM, Arthurs OJ (2016) Perinatal and paediatric post-mortem magnetic resonance imaging (PMMR): sequences and technique. Br J Radiol 89(1062): 20151028

4. Szafer A, Zhong J, Anderson AW, Gore JC (1995) Diffusionweighted imaging in tissues: theoretical models. NMR Biomed 8(7-8):289-296

5. Le Bihan D, Breton E, Lallemand D, Grenier P, Cabanis E, LavalJeantet M (1986) MR imaging of intravoxel incoherent motions: application to diffusion and perfusion in neurologic disorders. Radiology 161(2):401-407

6. Kocaoglu M, Bulakbasi N, Sanal HT, Kismet E, Caliskan B, Akgun V, Tayfun C (2010) Pediatric abdominal masses: diagnostic accuracy of diffusion weighted MRI. Magn Reson Imaging 28(5):629 636

7. Chavhan GB, Caro-Dominguez P (2016) Diffusion-weighted imaging in pediatric body magnetic resonance. Pediatr Radiol 46(6): 847-857

8. Morani AC, Smith EA, Ganeshan D, Dillman JR (2015) Diffusionweighted MRI in pediatric inflammatory bowel disease. AJR 204(6):1269-1277

9. TJP B, Vendhan K, Ambrose N, Atkinson D, Punwani S, Fisher C, Sen D, Ioannou Y, Hall-Craggs MA (2017) Diffusion-weighted imaging is a sensitive biomarker of response to biologic therapy in enthesitis related arthritis. Rheumatology 56(3):399-407

10. Genest DR, Williams MA, Greene MF (1992) Estimating the time of death in stillborn fetuses: I. Histologic evaluation of fetal organs; an autopsy study of150 stillborns. Obstet Gynecol 80:575-584

11. Papadopoulou I, Langan D, Sebire NJ, Jacques TS, Arthurs OJ (2016) Diffusion-weighted post-mortem magnetic resonance imaging of the human fetal brain in situ. Eur J Radiol 85:1167-1173

12. Arthurs OJ, Price GC, Carmichael DW, Jones R, Norman W, Taylor AM, Sebire NJ (2015) Diffusion-weighted perinatal postmortem magnetic resonance imaging as a marker of postmortem interval. Eur Radiol 25:1399-1406

13. Shepherd TM, Flint JJ, Thelwall PE, Stanisz GJ, Mareci TH, Yachnis AT, Blackband SJ (2009) Postmortem interval alters the water relaxation and diffusion properties of rat nervous tissueimplications for MRI studies of human autopsy samples. Neuroimage 44(3):820-826

14. Keller S, Schmidt TM, Kim AC, Fischer R, Heinemann A, Adam G, Yamamura J (2017) Postmortem MR diffusion-weighted imaging of the liver: time-behavior of the hepatic apparent diffusion coefficient in the early death interval. Int J Legal Med

15. Kang X, Cannie MM, Arthurs OJ, Segers V, Fourneau C, Bevilacqua E, Sanchez TC, Sebire NJ, Jani JC (2017) Postmortem whole-body magnetic resonance imaging of human fetuses: a comparison of 3-T vs. 1.5-T MR imaging with classical autopsy. Eur Radiol 27(8):3542-3553

16. Lavdas I, Miguel ME, McRobbie DW, Aboagye EO (2014) Comparison between diffusion-weighted MRI (DW-MRI) at 1.5 and 3 tesla: a phantom study. J Magn Reson Imaging 40(3):682690

17. Gold KJ, Abdul-Mumin AR, Boggs ME, Opare-Addo HS, Lieberman RW (2014) Assessment of 'fresh' versus 'macerated' as accurate markers of time since intrauterine fetal demise in lowincome countries. Int J Gynaecol Obstet 125(3):223-227 